\title{
Brucella abortus Induces Collagen Deposition and MMP-9 Down-Modulation in Hepatic Stellate Cells via TGF- $\beta 1$ Production
}

\author{
Paula C. Arriola Benitez, ${ }^{\star}$ Romina Scian, ${ }^{*}$ Diego J. Comerci, ${ }^{\dagger}$ Diego Rey Serantes, ${ }^{\dagger}$ Silvia Vanzulli, ${ }^{\dagger}$ Carlos A. Fossati, ${ }^{\S}$
} Guillermo H. Giambartolomei, ${ }^{*}$ and M. Victoria Delpino*

\begin{abstract}
From the Institute of Immunology, Genetics, and Metabolism, * Jose de San Martin Clinical Hospital, Faculty of Medicine and the Institute for the Study of Humoral Immunity, ${ }^{\S}$ Faculty of Pharmacy and Biochemistry, University of Buenos Aires, Buenos Aires; the Biotechnology Research Institute, ${ }^{\dagger}$ Technology Institute of Chascomús, National University of San Martin National Scientific and Technical Research, Buenos Aires; the Institute of Experimental Medicine, National Academy of Medicine, Buenos Aires, and the Laboratory of Immune System Research, ${ }^{\top}$ Faculty of Exact Sciences, National University of La Plata, La Plata, Argentina
\end{abstract}

Accepted for publication August 12, 2013.

Address correspondence to María Victoria Delpino, Ph.D. Instituto de Inmunología, Genética y Metabolismo, Hospital de Clínicas "José de San Martín," Facultad de Medicina, Universidad de Buenos Aires, Córdoba 2351 piso 3 sala 4 , 1120, Buenos Aires, Argentina. E-mail: mdelpino@ffyb.uba.ar.
In patients with active brucellosis, the liver is frequently affected by histopathologic lesions, such as granulomas, inflammatory infiltrations, and parenchymal necrosis. Herein, we examine some potential mechanisms of liver damage in brucellosis. We demonstrate that Brucella abortus infection inhibits matrix metalloproteinase-9 (MMP-9) secretion and induces collagen deposition and tissue inhibitor of matrix metalloproteinase- 1 secretion induced by hepatic stellate cells (LX-2). These phenomena depend on transforming growth factor- $\beta 1$ induction. In contrast, supernatants from $B$. abortus-infected hepatocytes and monocytes induce MMP-9 secretion and inhibit collagen deposition in hepatic stellate cells. Yet, if LX-2 cells are infected with $B$. abortus, the capacity of supernatants from $B$. abortus-infected hepatocytes and monocytes to induce MMP-9 secretion and inhibit collagen deposition is abrogated. These results indicate that depending on the balance between interacting cells and cytokines of the surrounding milieu, the response of LX-2 cells could be turned into an inflammatory or fibrogenic phenotype. Livers from mice infected with $B$. abortus displayed a fibrogenic phenotype with patches of collagen deposition and transforming growth factor- $\beta 1$ induction. This study provides potential mechanisms of liver immune response induced by $B$. abortus-infected hepatic stellate cells. In addition, these results demonstrate that the cross talk of these cells with hepatocytes and macrophages implements a series of interactions that may contribute to explaining some of mechanisms of liver damage observed in human brucellosis. (Am J Pathol 2013, 183: 1918-1927; http://dx.doi.org/10.1016/j.ajpath.2013.08.006)
Human brucellosis is a protean disease with a diversity of clinical signs and symptoms. ${ }^{1,2}$ It can affect almost any organ or system, causing focal forms that account for $30 \%$ of the reported cases. ${ }^{3}$

The liver is frequently affected in patients with active brucellosis. Clinical and biochemical features of liver involvement were found in up to $50 \%$ of patients with active disease. Yet, the most usual clinical manifestation of hepatic involvement is a mildly tender hepatomegaly. ${ }^{4}$ In a series of histopathologic studies of patients with brucellosis in which liver biopsy was performed, granulomas ranging from single parenchymal granulomatas to multiple localizations in portal space and parenchymal tissue were described. Most patients presented with inflammatory infiltrations, and half of them exhibited parenchymal necrosis. ${ }^{5}$ There also have been reports about a possible causal relationship between Brucella abortus infection and cirrhosis, which has not been definitively

Supported by grants PICT2010-0023 and PICT 2011-1501 from Agencia Nacional de Promoción Científica y Tecnológica, grant UBACYT 20020090200012 from Universidad de Buenos Aires, and grant PIP112200801-02706 from Consejo Nacional de Investigaciónes Científicas y Técnicas (CONICET) (all to M.V.D.). P.C.A.B., and R.S. are recipients of a fellowship from CONICET. D.J.C., C.A.F, G.H.G., and M.V.D. are members of the Research Career of CONICET.

The funders had no role in the study design, data collection and analysis, decision to publish, or preparation of the manuscript. 
established. ${ }^{5}$ Although numerous studies have focused on brucellar liver histopathology, the pathogenic mechanisms of liver disease caused by Brucella have not been completely investigated at the molecular and cellular levels.

The liver plays an important role in the innate immune response, providing the first line of defense against microbes and toxins crossing the intestinal barrier. ${ }^{6}$ Previously, we demonstrated that $B$. abortus-infected hepatocytes play a role in recruiting monocytes and neutrophils at the site of infection and in generating an inflammatory microenvironment with production of cytokines and matrix metalloproteinases (MMPs), all of which may mediate liver injury.

Resolution of inflammation of the liver tissues may proceed via the production of fibrogenic factors, such as transforming growth factor- $\beta 1$ (TGF- $\beta 1$ ). ${ }^{8}$ The activation of hepatic stellate cells is crucial in the healing of liver tissues because they are considered to be the major cell population that produces extracellular matrix components in the liver and because they play a pivotal role in its remodeling. ${ }^{9}$

Therefore, we hypothesized that liver inflammation induced by $B$. abortus infection ${ }^{5,7}$ may activate hepatic stellate cells to secrete type I collagen and profibrogenic cytokines, such as TGF- $\beta 1$ and IL-6, that would help in resolving the inflammatory process. To investigate this hypothesis, we used the human hepatic stellate cell line LX-2. In addition, the interaction among stellate cells, hepatocytes, and monocytes that could be attracted to the site of infection was also examined to determine the effect of this interaction on type I collagen deposition, MMP-9 production, and proinflammatory cytokine secretion.

\section{Materials and Methods}

\section{Bacterial Culture}

B. abortus $\mathrm{S} 2308$ was grown overnight in $10 \mathrm{~mL}$ of tryptic soy broth (Merck, Buenos Aires, Argentina) with constant agitation at $37^{\circ} \mathrm{C}$. Bacteria were harvested and the inocula were prepared as described previously. ${ }^{10}$ All live Brucella manipulations were performed in biosafety level 3 facilities located at the Instituto de Investigaciones Biomédicas en Retrovirus y SIDA (Buenos Aires, Argentina).

\section{Cell Culture}

The LX-2 cell line, a spontaneously immortalized human hepatic stellate cell line, was a gift from Dr. Scott L. Friedman (Mount Sinai School of Medicine, New York, NY). LX-2 cells were maintained in Dulbecco's modified Eagle's medium (Life Technologies-Invitrogen, Carlsbad, CA) and supplemented with $2 \mathrm{mmol} / \mathrm{L}$ L-glutamine, 100 $\mathrm{U} / \mathrm{mL}$ of penicillin, $100 \mu \mathrm{g} / \mathrm{mL}$ of streptomycin, and $2 \%$ (v/ v) fetal bovine serum (Gibco-Invitrogen, Carlsbad, CA). The human hepatoma cell line HepG2 and the human monocytic cell line THP-1 were obtained from the ATCC (Manassas, VA) and were cultured as previously described. ${ }^{7}$
To induce THP-1 maturation, cells were cultured in the presence of $0.05 \mu \mathrm{mol} / \mathrm{L} \mathrm{1,} \mathrm{25-dihydroxyvitamin} \mathrm{D3} \mathrm{(Cal-}$ biochem-Nova Biochem International, La Jolla, CA) for 72 hours. All the cultures were grown at $37^{\circ} \mathrm{C}$ and $5 \% \mathrm{CO}_{2}$.

\section{Cellular Infection}

LX-2 cells were infected with $B$. abortus at different multiplicities of infection (MOIs), HepG2 cells at MOI 1000, and THP-1 cells at MOI 100. After the bacterial suspension was dispensed, the plates were centrifuged for 10 minutes at $2000 \mathrm{rpm}$ and then were incubated for 2 hours at $37^{\circ} \mathrm{C}$ in a $5 \% \mathrm{CO}_{2}$ atmosphere. Cells were extensively washed with Dulbecco's modified Eagle's medium to remove extracellular bacteria and were incubated in medium supplemented with $100 \mu \mathrm{g} / \mathrm{mL}$ of gentamicin and $50 \mu \mathrm{g} / \mathrm{mL}$ of streptomycin to kill extracellular bacteria. LX-2 cells were harvested at different times to determine cytokine production, MMP secretion, $\alpha$-smooth muscle actin ( $\alpha$-SMA) expression, and collagen deposition. Supernatants from HepG2 and THP- 1 cells were harvested 24 hours after tinfection to be used as conditioned medium.

\section{Apoptosis Assays}

LX-2 cells were infected at different MOIs, and 24 hours after infection, cells were washed, and the percentage of apoptotic cells was assessed by the annexin V-fluorescein isothiocyanate assay (Sigma-Aldrich de Argentina SA, Buenos Aires, Argentina) using fluorescence-activated cell sorting. The percentage of apoptotic cells was also assessed by fluorescence microscopy after staining with Hoechst 33342 dye. As a positive control, cells were treated with $4 \%$ paraformaldehyde.

\section{Stimulation with Conditioned Media}

Culture supernatants from B. abortus-infected THP-1 monocytes and HepG2 hepatocytes were harvested 24 hours after infection, sterilized by filtration through a $0.22-\mu \mathrm{m}$ nitrocellulose filter, and used to stimulate infected and noninfected LX-2 cells. Supernatants were used diluted 1:2, 1:5, or 1:10 in complete medium. After 24 hours, the cells were harvested to determine MMPs and cytokine production or at 7 , 14 , or 30 days were assayed to determine collagen deposition by Sirius red staining.

\section{Zymography}

Gelatinase activity was assayed by the method of Hibbs et $\mathrm{al}^{10}$ with modifications, as described. ${ }^{11,12}$

\section{Measurement of Cytokine Concentrations}

Secretion of TGF- $\beta 1$, IL-6, IL-8, tumor necrosis factor $\alpha$, and monocyte chemotactic protein-1 in the supernatants was 
quantified by enzyme-linked immunosorbent assay (ELISA; BD Biosciences, San Jose, CA) in culture supernatants.

\section{Assessment of Collagen Deposition by Sirius Red Staining}

Collagen deposition was quantified using Sirius red (SigmaAldrich de Argentina SA), a strong anionic dye that binds strongly to collagen molecules. ${ }^{13}$ Sirius red was dissolved in saturated aqueous picric acid at a concentration of $0.1 \%$. Bouin fluid (for cell fixation) was prepared by mixing 15 $\mathrm{mL}$ of saturated aqueous picric acid with $5 \mathrm{~mL}$ of $35 \%$ formaldehyde and $1 \mathrm{~mL}$ of glacial acetic acid.

Cell layers were extensively washed with PBS before they were fixed with $1 \mathrm{~mL}$ of Bouin fluid for 1 hour. The fixation fluid was removed, and the culture plates were washed three times with deionizated water. The culture dishes were airdried before adding $1 \mathrm{~mL}$ of Sirius red dye reagent. The cells were stained for 18 hours under mild shaking. The stained cell layers were extensively washed with $0.01 \mathrm{~N}$ hydrochloric acid to remove all nonbound dye. After rinsing, coverslips were mounted in PBS-glycerine (9:1 [v/v]) and were analyzed by light microscopy. For quantitative analysis, the stained material was dissolved in $0.2 \mathrm{~mL}$ of $0.1 \mathrm{~N}$ sodium hydroxide by shaking for 30 minutes. The dye solution was transferred to microtiter plates, and the OD was measured using a microplate reader (Metertech Inc., Taipei, Taiwan) at $550 \mathrm{~nm}$ against $0.1 \mathrm{~N}$ sodium hydroxide as a blank.

\section{Immunofluorescence}

Infected LX-2 cells were fixed in 3\% paraformaldehyde for 10 minutes at room temperature and were permeabilized with $0.3 \%$ Triton X-100 (Roche Diagnostics GmbH, Mannheim, Germany) for 10 minutes. Cells were first incubated with rabbit anti- $\alpha$-SMA (Thermo Fisher Scientific Inc., Waltham, MA) diluted in PBS-Tween $0.1 \%$ for 30 minutes at room temperature, and then with rhodamine-conjugated anti-rabbit antibodies (Santa Cruz Biotechnology, Santa Cruz, CA). For collagen $\alpha 1$ (I) deposition, B. abortusinfected cells were fixed 7 days after infection and were subsequently incubated with mouse anti-collagen $\alpha 1$ (I) (Thermo Fisher Scientific Inc.) diluted in PBS-Tween $0.1 \%$ for 30 minutes at room temperature, and then with Alexa Fluor 488 anti-mouse antibodies (Invitrogen). DAPI was used for nuclear staining for 30 minutes at room temperature. After washing in PBS, cells were mounted and then were analyzed by fluorescence microscopy.

\section{Neutralization of TGF- $\beta 1$}

Neutralization experiments were performed with an antibody against human TGF- $\beta 1$ (IgG, rabbit polyclonal; Abcam Inc., Cambridge, MA) or its isotype control (rabbit IgG). To perform neutralizing experiments, anti-TGF- $\beta 1$ antibody was included during the infection at a final concentration of $20 \mu \mathrm{g} / \mathrm{mL}$.
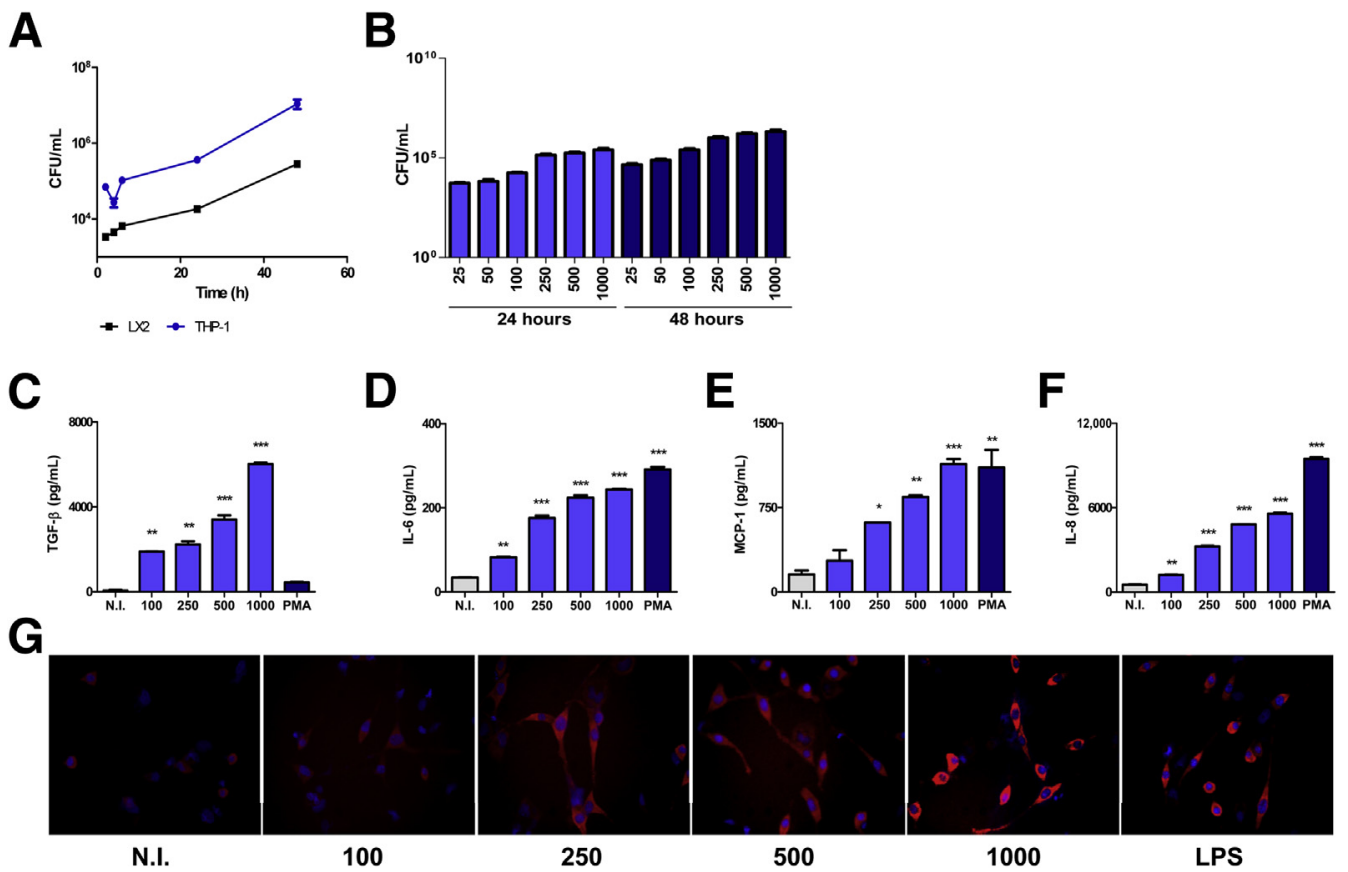

Figure $1 \quad B$. abortus invades and multiplies in LX-2 hepatic stellate cells, inducing profibrogenic activation. A: LX-2 and THP-1 cells were infected with B. abortus (MOI 100), and replication in each cell type was assessed by determining colony forming units (CFUs) after 2, 4, 6, 24, and 48 hours. B: Infection with $B$. abortus was performed at different MOIs, and CFUs were determined 24 and 48 hours after infection in LX-2 cells. ELISA determination of the cytokines TGF- $\beta 1$ (C) and IL-6 (D) and the chemokines monocyte chemotactic protein-1 (MCP-1) (E) and IL-8 (F) in culture supernatants of 24 hour-infected LX-2 cells. G: $\alpha$-SMA expression revealed by immunofluorescence with a specific antibody, Escherichia coli lipopolysaccharide (LPS), was used as a positive control. PMA, phorbol myristate acetate. Data are given as the means \pm SEM of duplicates. Data shown are from a representative experiment of three performed. ${ }^{*} P<0.1$, ${ }^{* *} P<0.01$, and ${ }^{* * * P}<0.001$ versus noninfected (N.I.). 


\section{Evaluation of Hepatic Fibrosis in a Mouse Model of Infection}

Six- to 8-week-old female BALB/c mice were infected through the i.p. route with $5 \times 10^{5}$ colony-forming units of B. abortus S2308 or vehicle (PBS). Mice were sacrificed 1, 4,8 , and 12 weeks after tinfection. To determine TGF- $\beta 1$ levels and collagen production in mouse livers, a liver lobe from each mouse was excised and placed immediately into 1 $\mathrm{mL}$ of cold PBS. Liver extractions were performed by using a tissue homogenizer. Homogenates were centrifuged at $2000 \times g$ for 20 minutes at $4^{\circ} \mathrm{C}$, and supernatants were stored at $-70^{\circ} \mathrm{C}$ until TGF- $\beta 1$ and collagen measurements were performed. In another group of mice, histologic examination of liver was performed 12 weeks after infection after routine fixation and paraffin embedding. Sections ( $5 \mu \mathrm{m}$ thick) were cut and stained with H\&E, Masson trichrome, and Sirius red stain. Masson trichrome staining was conducted according to the manufacturer's instructions. Briefly, sections were fixed in Bouin solution. After incubation in Weigert iron hematoxylin solution, the slides were stained with Biebrich scarlet-acid fuchsin and aniline blue and were dehydrated in ethanol and xylene. Extensive washes were done between each staining. Collagen fibers stain green or blue. Muscle and keratin are red. Cytoplasm is pink to red. Nuclei stain black. The Sirius red staining method was used to specifically stain fibrous tissue components. For Sirius red staining, sections were incubated for 30 minutes in $0.1 \%$ Sirius red $\mathrm{f} 3 \mathrm{~b}$ containing saturated picric acid and $0.1 \%$ fast green (Sigma-Aldrich de Argentina SA). After rinsing twice with distilled water, sections were briefly dehydrated using anhydrous alcohol and were coverslipped.

\section{Immunohistochemical Detection of TGF- $\beta 1$}

Immunohistochemical reactions were performed on sections of liver specimens prepared as described previously herein using monoclonal rabbit anti-mouse TGF- $\beta 1$ (Santa Cruz Biotechnology) diluted 1:200. After inhibition of the endogenous peroxidase with hydrogen peroxide in methanol and blockage of nonspecific binding, overnight incubation with the primary antibodies at $4^{\circ} \mathrm{C}$ in a humid chamber was performed. The next day, reactions were amplified using an EnVision+ dual link system horseradish peroxidase kit (Dako, Carpinteria, CA) and were developed using 3,3'diaminobenzidine and hydrogen peroxide (Sigma-Aldrich de Argentina SA). Nuclear counterstaining was performed using hematoxylin.

\section{Statistical Analysis}

Statistical analysis was performed using one-way analysis of variance, followed by post hoc Tukey testing using GraphPad Prism version 4.0 software (GraphPad Software Inc., San Diego, CA). Data are presented as means \pm SEM.

\section{Results}

B. abortus Invades and Multiplies in LX-2 Cells, Inducing Profibrogenic Activation

Hepatic stellate cells clearly have a necessary and fundamental role in tissue homeostasis and normal wound repair through the production of extracellular matrix proteins. ${ }^{14}$ The persistence of an infectious stimulus might drive liver fibrosis because its presence could induce marked alterations in a variety of immune and structural cells.

To begin to substantiate the hypothesis, LX-2 cells were infected with $B$. abortus. The bacterium invades and replicates in LX-2 cells, and the magnitude of the infection (intracellular colony-forming units) was directly related to the MOI used (Figure 1, A and B). As a control, THP-1 cells, a monocytic/macrophage cell line that was consistently reported to support Brucella infection and growth, were infected in parallel. At any time tested, the number of bacteria was higher in THP-1 cells than in LX-2 cells (Figure 1A). This is a consistent result because it has been
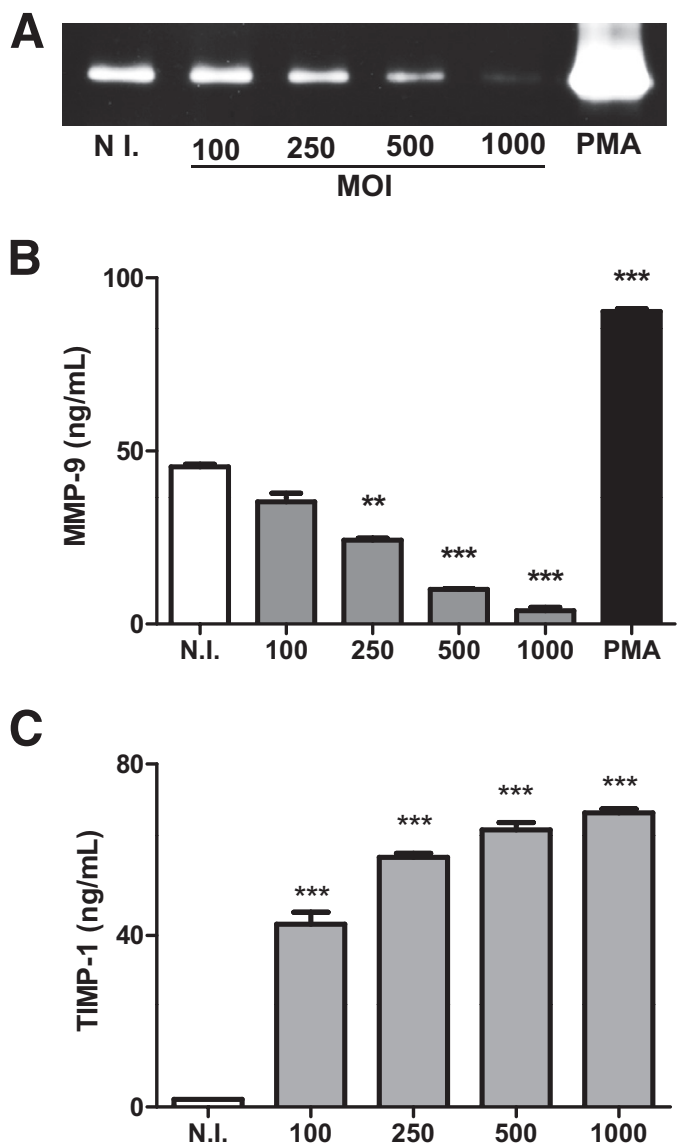

Figure 2 B. abortus infection inhibits MMP-9 secretion and induces TIMP-1 production. LX-2 hepatic stellate cells were infected with $B$. abortus, and 24 hours after infection, supernatants were harvested to analyze MMP-9 production by zymography (A) and ELISA (B) and TIMP-1 production by ELISA (C). PMA, phorbol myristate acetate. Data are given as the means \pm SEM of duplicates. Data shown are from a representative experiment of three performed. ${ }^{* *} P<0.01,{ }^{* \star *} P<0.001$ versus noninfected (N.I.). 
A
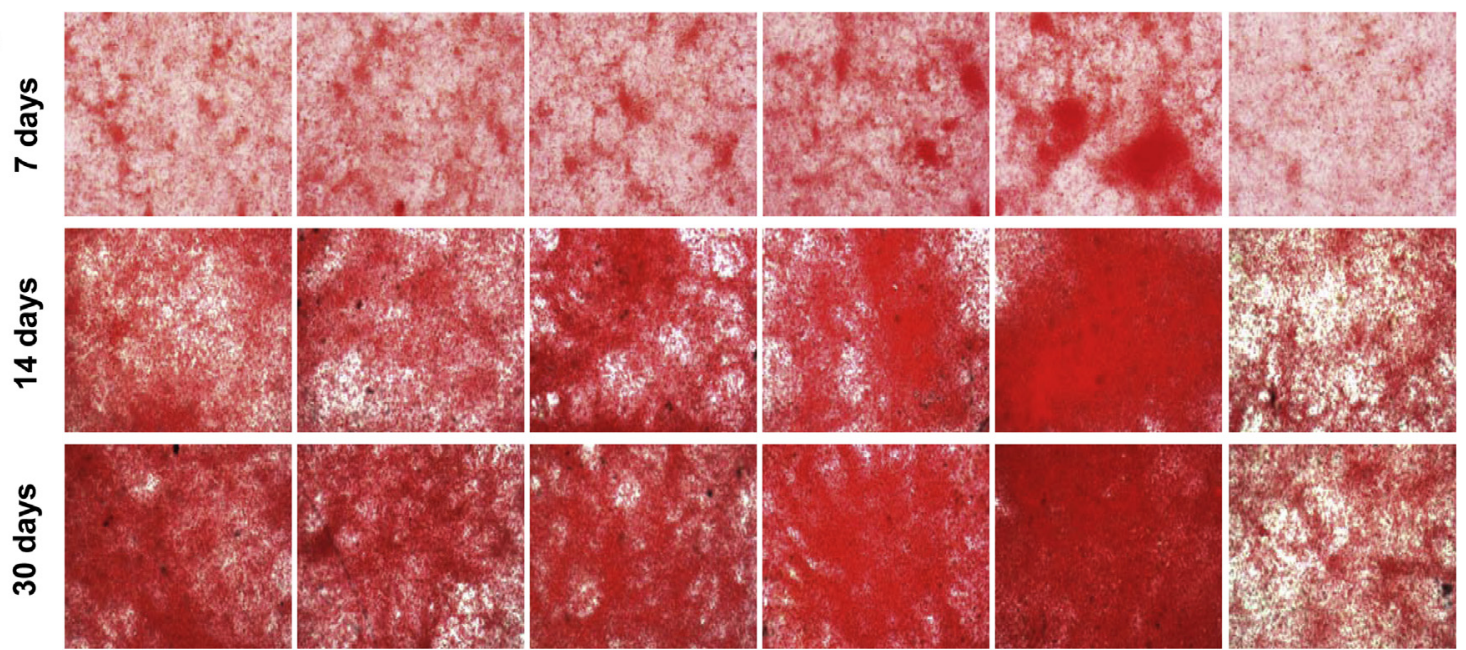

N.I.
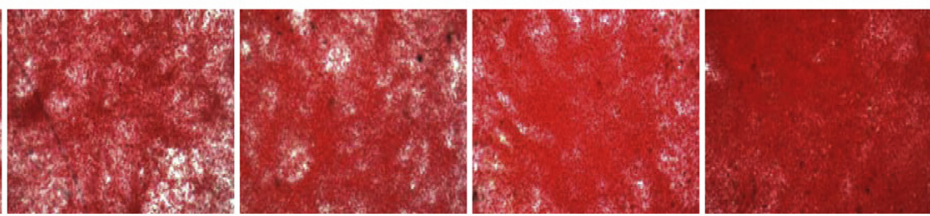

1000

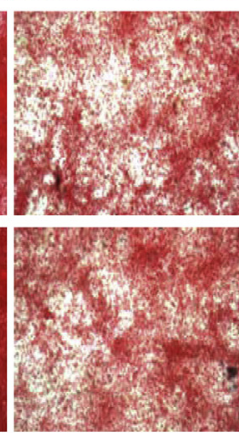

100

250

500

14 days

30 days
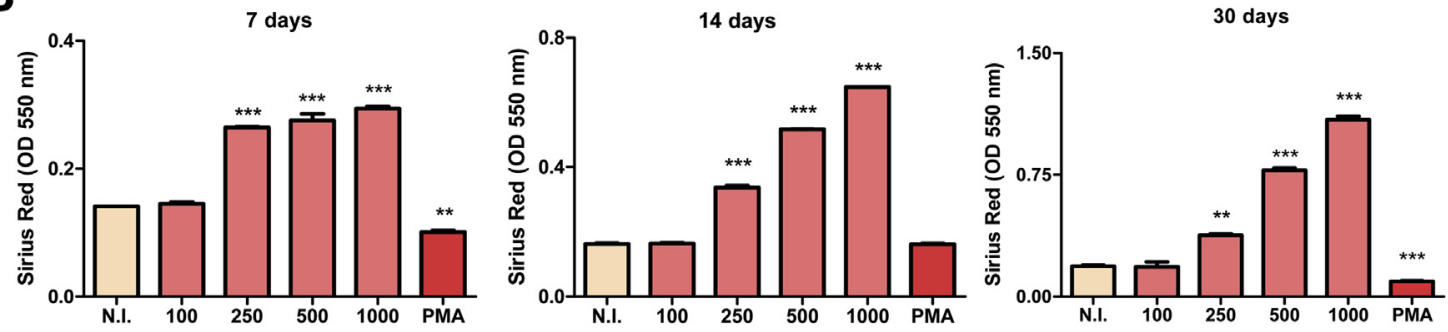

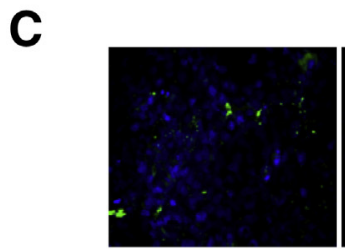

N.I.

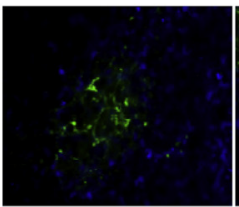

100

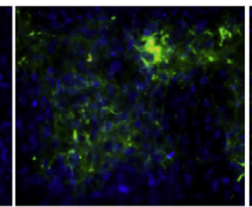

250

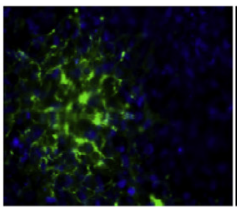

500

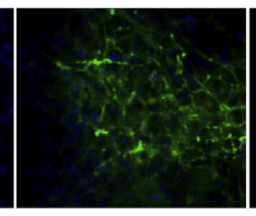

1000

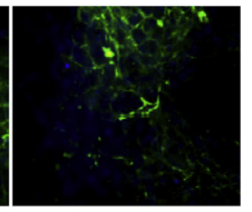

PMA

Figure 3 LX-2 hepatic stellate cells infected with B. abortus induce collagen deposition. A: At 7, 14, and 30 days of LX-2 cell cultures, collagen deposition was revealed by Sirius red staining. B: Quantification of Sirius red was determined by OD readings at $550 \mathrm{~nm}$. C: Collagen deposition was revealed by immunofluorescence with a specific antibody. PMA, phorbol myristate acetate. Data are given as the means \pm SEM of duplicates. Data shown are from a representative experiment of three performed. ${ }^{* *} P<0.01,{ }^{* * *} P<0.001$ versus noninfected (N.I.).

reported that macrophages are the preferential cells that support Brucella replication. ${ }^{15}$

To determine whether $B$. abortus infection affects the viability of LX-2 cells, these cells were infected with B. abortus for 2 hours and then were washed to remove uninternalized bacteria; after 24 hours, nuclei were stained with Hoechst 33342 and were analyzed by microscopy, or cells were stained with annexin V-phosphatidylinositol and were analyzed by flow cytometry. Paraformaldehyde at $4 \%$ was used as a positive control of apoptosis. B. abortus infection did not induce apoptosis of LX-2 cells at any MOI tested as measured by Hoechst staining or by annexin V-phosphatidylinositol staining (data not shown). These results are consistent with a putative fibrotic phenotype induced by LX-2 cells because their death would impede the healing of hepatic tissues, modulating the hepatic microenvironment toward an antifibrogenic balance. ${ }^{16}$
Infection also resulted in significant secretion of the profibrogenic cytokines TGF- $\beta 1$ and IL- 6 and the chemokines monocyte chemotactic protein-1 and IL-8 in an MOIdependent manner but not tumor necrosis factor $\alpha$ (Figure 1, $\mathrm{C}-\mathrm{F}$, and data not shown).

Because $B$. abortus induced the secretion of profibrogenic cytokines (TGF- $\beta$ and IL-6), experiments were conducted to determine whether $B$. abortus infection could also induce an increase in $\alpha$-SMA expression, another marker of myofibroblast differentiation and activation of fibrogenesis. To this end, LX-2 cells were infected with $B$. abortus, and after 72 hours, $\alpha$-SMA production was detected using a specific antibody. Lipopolysaccharide from Escherichia coli was used as a positive control. B. abortus infection induced an $\alpha$-SMA increase in an MOI-dependent manner (Figure 1G). 


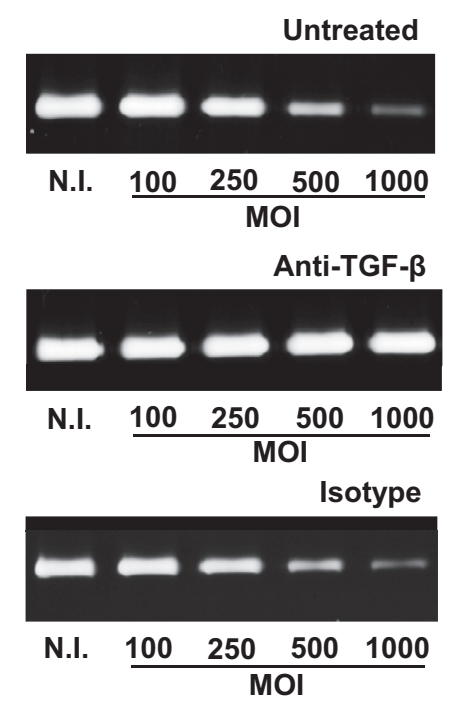

Figure 4 B. abortus inhibition of MMP-9 activity depends on TGF- $\beta 1$ secretion. Inhibition of MMP-9 secretion by $B$. abortus-infected LX-2 hepatic stellate cells in the presence of an anti-TGF- $\beta 1$ neutralizing antibody or its isotype control. Data shown are from a representative experiment of three performed.

Taken together, these results indicate that $B$. abortus infection induces LX-2 cell activation, which leads to concomitant profibrogenic phenotype.

\section{B. abortus Inhibits MMP-9 Secretion and Concomitantly Induces TIMP-1 Secretion in LX-2 Cells}

The participation of MMPs and their specific inhibitors, the TIMPs, together with collagen deposition are implicated in the formation and recovery processes of liver fibrosis and granuloma formation. ${ }^{14}$ Therefore, experiments were conducted to determine whether $B$. abortus infection may modify the expression of these molecules. B. abortus infection reduced the basal levels of MMP-9 secreted by LX-2 in an MOI-dependent manner, as determined by gelatin zymography (Figure 2A) and corroborated by ELISA (Figure 2B). In contrast, significantly increased levels of TIMP-1 were detected in supernatants from infected LX-2 cells (Figure 2C). The magnitude of TIMP-1 release was directly related to the MOI used.

\section{LX-2 Cells Infected with $B$. abortus Induce Collagen Deposition}

Activated stellate cells could also display increased production of extracellular matrix and profibrotic factors such as collagen. Therefore, experiments were conducted to determine whether $B$. abortus infection induced collagen deposition. Collagen was stained by adding Sirius red, a strong anionic dye that binds strongly to collagen molecules. B. abortus-infected and noninfected LX-2 cells progressively deposited more collagen with time, but infected cells produced significantly $(P<0.01)$ more collagen than noninfected controls in an MOI-dependent manner on days 7 , 14, and 30 (Figure 3, A and B), demonstrating the stimulation of collagen deposition induced by $B$. abortus infection. To corroborate whether $B$. abortus infection may induce an increase of collagen I deposition, LX-2 cells were infected, and after 7 days, collagen $\alpha 1$ (I) deposition was detected with a specific antibody. Confirming the results obtained with Sirius red, B. abortus infection increased collagen I deposition in an MOI-dependent manner (Figure 3C). Taken together, these results indicate that $B$. abortus infection activates hepatic stellate cells, inducing collagen deposition. This deposition could induce progressive changes in the surrounding extracellular matrix, contributing to fibrogenesis.

\section{B. abortus Inhibition of MMP-9 Activity Depends on TGF- $\beta 1$ Secretion}

A key cytokine involved in the fibrotic phenotype from paracrine and autocrine sources is TGF- $\beta 1$, which is expressed by several cell types, including hepatic stellate cells. ${ }^{17}$ B. abortus infection stimulated TGF- $\beta 1$ secretion in LX-2 cells. Then, experiments were conducted to investigate whether TGF- $\beta 1$ was involved in MMP-9 regulation by $B$. abortus infection. To address this issue, we evaluated the
A

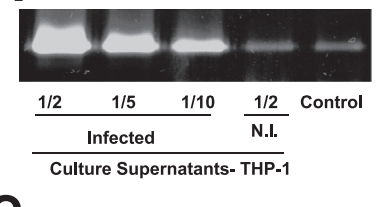

C

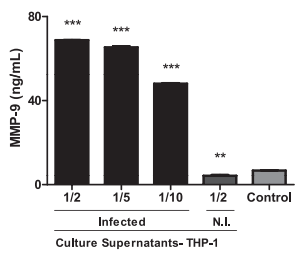

$\mathbf{E}$

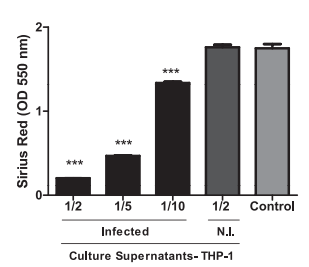

B

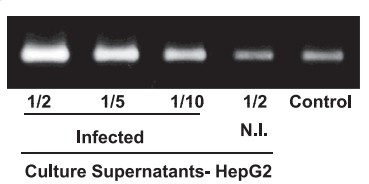

D

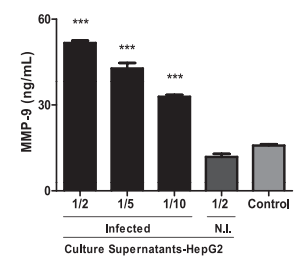

$\mathbf{F}$

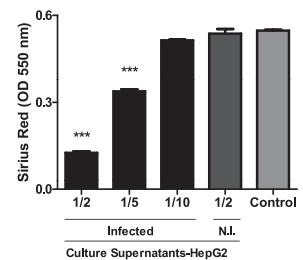

Figure 5 Supernatants from B. abortus-infected hepatocytes (HepG2) and monocytes (THP-1) induce MMP-9 secretion and inhibit collagen deposition by LX-2 hepatic stellate cells. MMP-9 production by noninfected (N.I.) LX-2 cells stimulated with supernatants from $B$. abortus-infected monocytes or hepatocytes (added at a $1: 2,1: 5$, or $1: 10$ proportion) or from noninfected monocytes or hepatocytes was determined by zymography (A and $\mathbf{B}$ ) and ELISA (C and D). E and F: Collagen production by N.I. LX-2 cells stimulated with supernatants from $B$. abortus-infected monocytes or hepatocytes was determined by quantification of Sirius red 7 days after stimulation. Data are given as the means \pm SEM of duplicates. Data shown are from a representative experiment of three performed. ${ }^{* *} P<0.01$, ${ }^{* * *} P<0.001$ versus control (nonstimulated). 
effects of neutralizing the action of this cytokine by using a TGF- $\beta 1$ neutralizing antibody. As shown in Figure 4, neutralization of TGF- $\beta 1$ reduced the ability of $B$. abortus to inhibit MMP-9 production in LX-2 cells. Isotype control had no effect. These results indicate that TGF- $\beta 1$ could be a key cytokine involved in the fibrogenic phenotype triggered by $B$. abortus infection.

\section{Supernatants from B. abortus-Infected Hepatocytes and Monocytes Induce MMP-9 Secretion and Inhibit Collagen Deposition by LX-2 Cells}

The liver is an intricate microenvironment in which diverse cell types (resident and infiltrating) can interact in a complex network. In view of the ability of Brucella-infected LX-2 cells to produce monocyte-attracting factors that could potentially recruit monocytes to the site of infection, we hypothesized that macrophages could be attracted to the site of infection. Thus, we decided to investigate the effect of cytokines present in supernatants from B. abortus-infected macrophages on MMP-9 production by LX-2 cells. The addition of supernatants from $B$. abortus-infected monocytes at different proportions (1:2 to $1: 10)$ to noninfected LX-2 cells induced significant secretion of MMP-9 by the latter cells compared with that in unstimulated cultures as determined by zymography and corroborated by ELISA. In contrast, MMP-9 secretion was not induced when LX-2 cells were stimulated with supernatants from noninfected monocytes (Figure 5, A and C).

Because we previously demonstrated that hepatocytes could be infected by B. abortus and that this infection elicited the secretion of cytokines, ${ }^{7}$ we decided to investigate whether supernatants from B. abortus-infected hepatocytes induced MMP-9 secretion by LX-2 cells. As shown in Figure 5, B and $\mathrm{D}$, supernatants from B. abortus-infected hepatocytes induced MMP-9 secretion by LX-2 cells in a dose-dependent manner. Supernatants from noninfected hepatocytes had no effect. In addition, the increase in MMP-9 secretion by LX-2 cells induced by supernatants from $B$. abortus-infected hepatocytes and monocytes is in correlation with an inhibition in collagen deposition as determined by Sirius red staining (Figure 5, E and F). These results indicate that supernatants from B. abortus-infected monocytes and hepatocytes induced MMP-9 secretion and inhibited collagen deposition by LX-2 cells.

However, these results also indicated that $B$. abortus infection of LX-2 cells inhibits basal MMP-9 secretion and induces collagen deposition. Thus, experiments were conducted to determine whether $B$. abortus infection may also inhibit MMP9 secretion induced by monocytes and hepatocytes, secreted factors with concomitant collagen deposition induction. For this, LX-2 cells were infected with B. abortus at different MOIs in the presence of supernatants from B. abortus-infected monocytes or hepatocytes. Supernatants from noninfected monocytes and hepatocytes were used as control. B. abortus infection of LX-2 cells inhibited MMP-9 secretion induced by supernatants from $B$. abortus-infected monocytes or hepatocytes (Figure 6, A and B). Concomitantly, B. abortus infection also induced collagen deposition induced in the presence of supernatants from $B$. abortus-infected monocytes or hepatocytes (Figure 6, C and D). These results indicate that depending on the status of LX-2 cells (infected or not) and the interaction with surrounding or attracted cells, it can induce a fibrotic or an inflammatory phenotype.

\section{LX-2 Cells Secrete TGF- $\beta 1$ in Response to $B$. abortus Infection and Stimulation with Supernatants from B. abortus-Infected Monocytes and Hepatocytes}

TGF- $\beta 1$ plays a pivotal role in the development of fibrosis in the liver and other organs. ${ }^{18-21}$ In addition, TGF- $\beta 1$ modulates

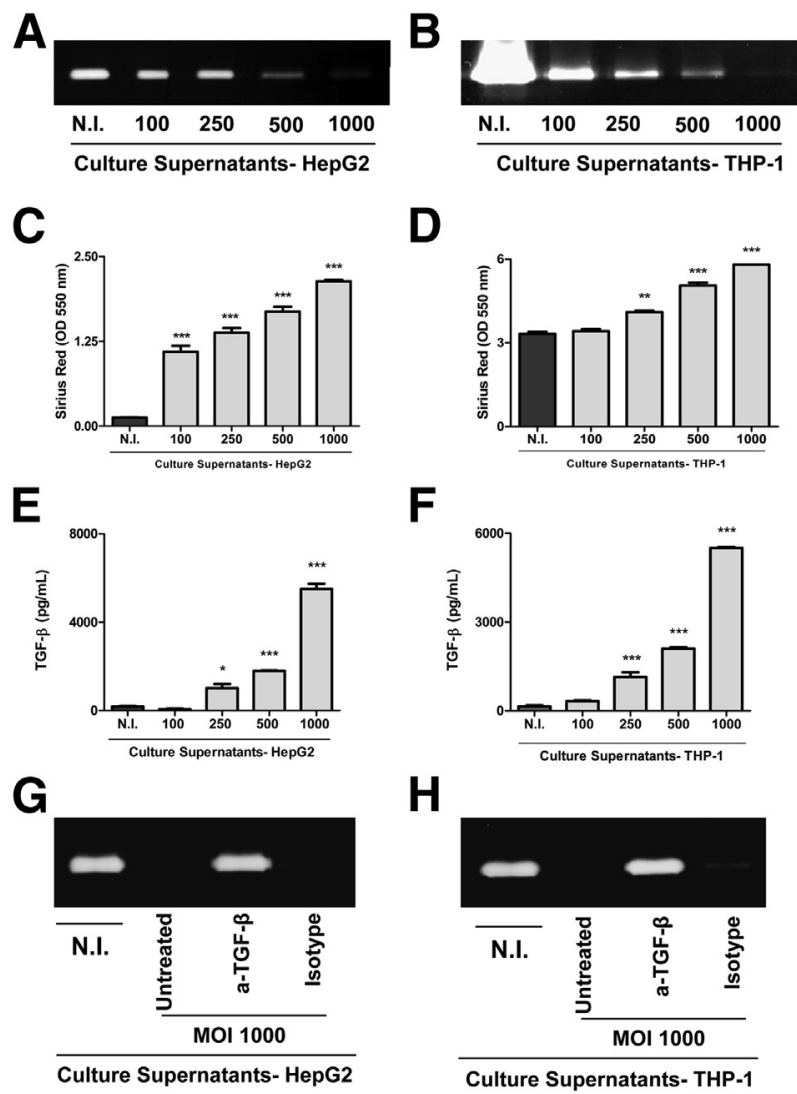

Figure $6 \quad B$. abortus infection of LX-2 cells inhibits MMP-9 secretion induced by supernatants from $B$. abortus-infected hepatocytes (HepG2) and monocytes (THP-1) while inducing collagen deposition. $\mathbf{A}$ and $\mathbf{B}$ : MMP-9 production by $B$. abortus-infected (MOI 100) LX-2 hepatic stellate cells stimulated with supernatants from $B$. abortus-infected hepatocytes or monocytes (added at a 1:2, 1:5, or 1:10 proportion) or from noninfected hepatocytes or monocytes was determined by zymography 24 hours after stimulation. $\mathbf{C}$ and $\mathbf{D}$ : Collagen production was determined by quantification of Sirius red 7 days after stimulation. $\mathbf{E}$ and $\mathbf{F}$ : TGF- $\beta 1$ secretion was measured by ELISA 24 hours after stimulation. $\mathbf{G}$ and $\mathbf{H}$ : Inhibition of the stimulating effect of TGF- $\beta 1$ by performing the experiments with an anti-TGF- $\beta 1$ neutralizing antibody or an isotype control. Data are given as the means \pm SEM of duplicates. Data shown are from a representative experiment of three performed. ${ }^{*} P<0.1,{ }^{*} P<0.01$, and ${ }^{* * *} P<0.001$ versus noninfected (N.I.). 

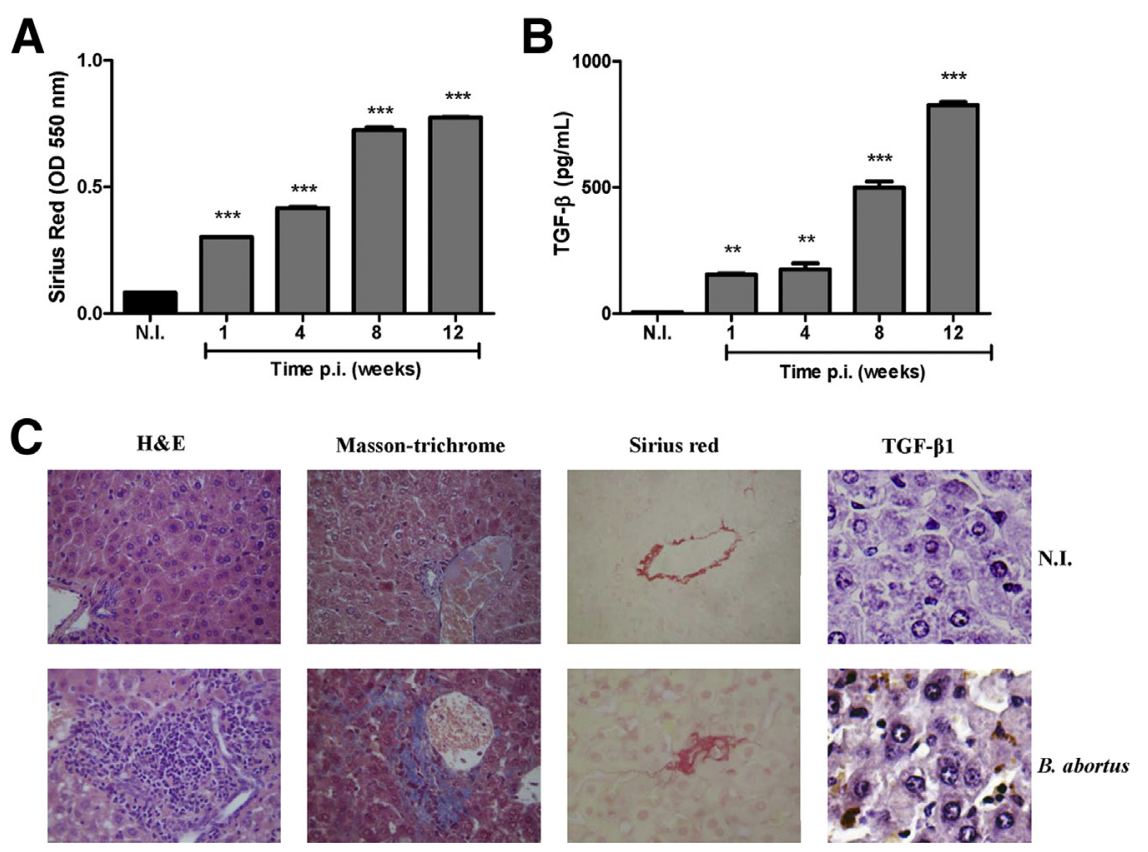

Figure 7 Immunopathologic markers in livers of mice infected with $B$. abortus. A: Collagen production was determined by quantification of Sirius red in liver extracts $1,4,8$, and 12 weeks after infection. B: TGF- $\beta 1$ secretion was measured by ELISA in liver extracts $1,4,8$, and 12 weeks after infection. C: Representative photomicrographs of liver sections from noninfected (N.I.) or $B$. abortus-infected mice $(n=6)$ stained with $\mathrm{H} \& \mathrm{E}$, Masson trichrome, Sirius red, and immunohistochemical staining for TGF- $\beta 1$. ${ }^{* *} P<0.01$, $* * * P<0.001$ versus N.I. the expression of TIMPs, MMPs, and collagen, among others. $^{22}$ Then, experiments were conducted to determine the role of this cytokine in the inhibition of MMP-9 secretion in B. abortus-infected LX-2 cells treated with supernatants from B. abortus - infected monocytes or hepatocytes. TGF- $\beta 1$ secretion was detected in culture supernatants from $B$. abortus-infected LX-2 cells that were treated with supernatants from B. abortus -infected monocytes or hepatocytes (Figure 6, $\mathrm{E}$ and F). However, this cytokine was not detected in supernatants from noninfected LX-2 cells treated with supernatants from B. abortus-infected monocytes or hepatocytes. To determine whether TGF- $\beta 1$ is involved in the inhibition of MMP-9 secretion, we performed the same experiment but in the presence of a neutralizing antibody anti-TGF- $\beta 1$. Inhibition of TGF- $\beta 1$ reverses the inhibitory effect induced by $B$. abortus infection; isotype control had no effect (Figure 6, G and $\mathrm{H})$.

Altogether, these results indicate that $B$. abortus infection prevents MMP-9 secretion induced by supernatants from B. abortus-infected monocytes and hepatocytes in a way in which TGF- $\beta 1$ is the key cytokine involved.

\section{Immunopathologic Markers in Livers of Mice Infected with $B$. abortus}

The hypothesis is that $B$. abortus could induce and inhibit MMP-9 secretion depending on cell-to-cell interaction and whether they were previously infected. As shown in Figure 7A, levels of collagen, measured by Sirius red staining, were significantly increased in the livers of mice infected with $B$. abortus compared with the livers of mice inoculated with PBS (negative control). In addition, this increase correlated with the increases in TGF- $\beta 1$ secretion, especially from week 8 on (Figure $7 \mathrm{~B}$ ). In addition, the histologic findings in liver sections after H\&E staining indicated that $B$. abortus infection induced an inflammatory lymphocytic infiltrate. Also, Masson trichrome and Sirius red staining revealed the presence of fibrotic patch and collagen deposition, respectively, in livers from B. abortusinfected mice (Figure 7C). Immunohistochemical analysis revealed that TGF- $\beta 1$ was mainly produced by hepatic stellate cells (Figure 7C).

\section{Discussion}

In patients with active brucellosis, the liver is frequently affected by histopathologic lesions, such as granulomas, inflammatory infiltrations, and parenchymal necrosis. ${ }^{5}$ In the present study, we examined some potential mechanisms of liver damage in brucellosis. Although numerous studies have focused on brucellar liver histopatology, ${ }^{5}$ consensus on the nature of this involvement is missing.

Liver fibrosis is characterized by the deposition of extracellular matrix. The persistence of an infectious stimulus, such as B. abortus infection, and the sustained injury might drive fibrosis because its presence induces marked alterations in a variety of immune and structural cells. ${ }^{23}$ Many potentially fibrogenic cell populations in the liver have been described, such as portal fibroblast, mesenchymal cells derived from the bone marrow, and even hepatocytes and biliary epithelial cells. ${ }^{24}$ However, hepatic stellate cells are recognized as the major source of liver fibrosis. ${ }^{25}$

Collagen type $\mathrm{I}$ is the prototype constituent of the fibrilforming matrix in fibrotic liver, and its expression is regulated at the transcriptional and posttranscriptional levels, as described. ${ }^{26-28}$ TGF- $\beta 1$, derived from paracrine and autocrine sources, remains the classic fibrogenic cytokine. ${ }^{29,30}$ We demonstrated that hepatic stellate cells (LX-2) infected 
with $B$. abortus increased the expression of $\alpha$-SMA, a key marker of stellate cell activation, collagen, and TGF- $\beta 1$ secretion. In correlation with these findings, B. abortus infection inhibited the basal and induced secretion of MMP-9 by hepatic stellate cells that was dependent on TGF- $\beta 1$ induction. The most frequent abnormalities in liver brucellosis are raised aminotransferase and alkaline phosphatase levels. ${ }^{4}$ A significant correlation between aspartate aminotransferase and alanine aminotransferase activity in serum and increased levels of TGF- $\beta 1$ has been previously reported. ${ }^{31}$ On the other hand, elevated concentrations of TGF- $\beta 1$ correlated with the severity of liver diseases, suggesting TGF- $\beta 1$ as a marker of hepatic dysfunction ${ }^{32}$ and possibly of hepatic fibrosis progression. ${ }^{3}$

In addition, we demonstrated that $B$. abortus-infected hepatic stellate cells not only contribute to collagen deposition but also may mediate the recruitment of inflammatory cells to the infectious focus. Consistent with this hypothesis, we found that LX-2 cells respond to B. abortus infection with significant production of IL-8 and monocyte chemotactic protein-1, potent chemoattractants for neutrophils and monocytes, respectively. This is particularly relevant because monocytes/macrophages are the preferential niche of replication of the bacterium. ${ }^{15}$ Therefore, macrophages (the ones that dwell in the liver or the ones that could be attracted to the site of infection) also could contribute to liver abnormalities, not only providing a replicative niche but also causing inflammation. Moreover, a recent study shed light on the complexity and dynamics of the cellular environment caused by this pathogen during the course of liver infection and demonstrated that hepatic dendritic cells are effectors and reservoir cells in chronicity of infection. ${ }^{34}$ However, although stellate cells could not be a replicative niche or Brucella reservoir in the liver, these cells could be infected transiently or stimulated by Brucella antigens or by cytokines present in the inflammatory milieu generated by the infection. Different studies ${ }^{35-37}$ revealed the intracellular signaling cascades involved in the Toll-like receptorinitiated immune response to Brucella spp. infection. A recent study showed the role of non-Toll-like receptors in innate immunity in Brucella infection, which results in activation of an acute inflammatory response that is partly mediated by a cytosolic machinery termed inflammasome. ${ }^{36}$ This very acute inflammatory pathway is also vital for the development of a full fibrogenic response in a variety of organs, including the lungs, liver, and skin. ${ }^{38}$

Accordingly, we found that supernatants from B. abortusinfected macrophages induced MMP-9 secretion and inhibited collagen deposition by LX-2 cells; however, these phenomena were inhibited when LX-2 cells were infected with $B$. abortus at the same time that they were treated with supernatants from B. abortus-infected macrophages. This also correlated with an increase in TGF- $\beta 1$ production. The same phenomena were observed when we used supernatants from B. abortus-infected hepatocytes. These results and previous findings ${ }^{7}$ correlated with the histologic findings from the liver of $B$. abortus-infected mice, in which we observed inflammatory damage and fibrotic regions simultaneously. In addition, in liver extracts from infected mice we observed a significant increase in collagen deposition and TGF- $\beta 1$ secretion in a time-dependent manner. Histologic analysis revealed the presence of inflammatory infiltrate and patched fibrosis.

In summary, the present study provides potential mechanisms of liver fibrosis induced by $B$. abortus -infected hepatic stellate cells. In addition, the cross talk of these cells with hepatocytes and macrophages would put into action a series of interactions that may contribute to explaining some of mechanisms of liver damage observed in human brucellosis.

\section{Acknowledgments}

We thank Horacio Salomón and the staff of the Instituto de Investigaciones Biomédicas en Retrovirus y Sida for their assistance with biosafety level 3 laboratory use.

\section{References}

1. Young EJ: An overview of human brucellosis. Clin Infect Dis 1995, 21:283-289

2. Pappas G, Akritidis N, Bosilkovski M, Tsianos E: Brucellosis. N Engl J Med 2005, 352:2325-2336

3. Colmenero JD, Reguera JM, Martos F, Sanchez-De-Mora D, Delgado M, Causse M, Martin-Farfan A, Juarez C: Complications associated with Brucella melitensis infection: a study of 530 cases. Medicine (Baltimore) 1996, 75:195-211

4. Madkour MM: Gastrointestinal brucellosis. Madkour's Brucellosis. Edited by Madkour MM, ed 2. Berlin, Springer-Verlag, 2001, pp $150-158$

5. Akritidis N, Tzivras M, Delladetsima I, Stefanaki S, Moutsopoulos HM, Pappas G: The liver in brucellosis. Clin Gastroenterol Hepatol 2007, 5: 1109-1112

6. Crispe IN: The liver as a lymphoid organ. Annu Rev Immunol 2009, 27:147-163

7. Delpino MV, Barrionuevo P, Scian R, Fossati CA, Baldi PC: Brucellainfected hepatocytes mediate potentially tissue-damaging immune responses. J Hepatol 2010, 53:145-154

8. Friedman SL: Liver fibrosis: from bench to bedside. J Hepatol 2003, 38(Supp1 1):S38-S53

9. Sato M, Suzuki S, Senoo H: Hepatic stellate cells: unique characteristics in cell biology and phenotype. Cell Struct Funct 2003, 28:105-112

10. Hibbs MS, Hasty KA, Seyer JM, Kang AH, Mainardi CL: Biochemical and immunological characterization of the secreted forms of human neutrophil gelatinase. J Biol Chem 1985, 260:2493-2500

11. Scian R, Barrionuevo P, Giambartolomei GH, De Simone EA, Vanzulli SI, Fossati CA, Baldi PC, Delpino MV: Potential role of fibroblast-like synoviocytes in joint damage induced by Brucella abortus infection through production and induction of matrix metalloproteinases. Infect Immun 2011, 79:3619-3632

12. Scian R, Barrionuevo P, Giambartolomei GH, Fossati CA, Baldi PC, Delpino MV: Granulocyte-macrophage colony-stimulating factor- and tumor necrosis factor alpha-mediated matrix metalloproteinase production by human osteoblasts and monocytes after infection with Brucella abortus. Infect Immun 2011, 79:192-202

13. Tullberg-Reinert H, Jundt G: In situ measurement of collagen synthesis by human bone cells with a sirius red-based colorimetric microassay: effects of transforming growth factor beta2 and ascorbic acid 2-phosphate. Histochem Cell Biol 1999, 112:271-276 
14. Flamm SL: Granulomatous liver disease. Clin Liver Dis 2013, 16:387-396

15. Roop RM II, Bellaire BH, Valderas MW, Cardelli JA: Adaptation of the Brucellae to their intracellular niche. Mol Microbiol 2004, 52:621-630

16. Kotake S, Sato K, Kim KJ, Takahashi N, Udagawa N, Nakamura I, Yamaguchi A, Kishimoto T, Suda T, Kashiwazaki S: Interleukin-6 and soluble interleukin-6 receptors in the synovial fluids from rheumatoid arthritis patients are responsible for osteoclast-like cell formation. J Bone Miner Res 1996, 11:88-95

17. Friedman SL: Hepatic stellate cells: protean, multifunctional, and enigmatic cells of the liver. Physiol Rev 2008, 88:125-172

18. Friedman SL, Yamasaki G, Wong L: Modulation of transforming growth factor beta receptors of rat lipocytes during the hepatic wound healing response: enhanced binding and reduced gene expression accompany cellular activation in culture and in vivo. J Biol Chem 1994, 269:10551-10558

19. Jones CL, Buch S, Post M, McCulloch L, Liu E, Eddy AA: Renal extracellular matrix accumulation in acute puromycin aminonucleoside nephrosis in rats. Am J Pathol 1992, 141:1381-1396

20. Desmouliere A, Geinoz A, Gabbiani F, Gabbiani G: Transforming growth factor-beta 1 induces alpha-smooth muscle actin expression in granulation tissue myofibroblasts and in quiescent and growing cultured fibroblasts. J Cell Biol 1993, 122:103-111

21. Krieg T, Braun-Falco O: Fibrocytes and fibroblasts: definition and significance in wound healing and fibrotic diseases of the skin [in German]. Z Hautkr 1989, 64:775-776, 779-781

22. Shek FW, Benyon RC, Walker FM, McCrudden PR, Pender SL, Williams EJ, Johnson PA, Johnson CD, Bateman AC, Fine DR, Iredale JP: Expression of transforming growth factor-beta 1 by pancreatic stellate cells and its implications for matrix secretion and turnover in chronic pancreatitis. Am J Pathol 2002, 160:1787-1798

23. Meneghin A, Hogaboam CM: Infectious disease, the innate immune response, and fibrosis. J Clin Invest 2007, 117:530-538

24. Kalluri R, Neilson EG: Epithelial-mesenchymal transition and its implications for fibrosis. J Clin Invest 2003, 112:1776-1784

25. Liu Y, Meyer C, Muller A, Herweck F, Li Q, Mullenbach R, Mertens PR, Dooley S, Weng HL: IL-13 induces connective tissue growth factor in rat hepatic stellate cells via TGF-beta-independent Smad signaling. J Immunol 2011, 187:2814-2823

26. Arthur MJ: Reversibility of liver fibrosis and cirrhosis following treatment for hepatitis C. Gastroenterology 2002, 122:1525-1528

27. Issa R, Zhou X, Constandinou CM, Fallowfield J, Millward-Sadler H, Gaca MD, Sands E, Suliman I, Trim N, Knorr A, Arthur MJ, Benyon RC, Iredale JP: Spontaneous recovery from micronodular cirrhosis: evidence for incomplete resolution associated with matrix cross-linking. Gastroenterology 2004, 126:1795-1808

28. Pares A, Caballeria J, Bruguera M, Torres M, Rodes J: Histological course of alcoholic hepatitis: influence of abstinence, sex and extent of hepatic damage. J Hepatol 1986, 2:33-42

29. Inagaki $\mathrm{M}$, Wang Z, Carr BI: Transforming growth factor beta 1 selectively increases gene expression of the serine/threonine kinase receptor 1 (SKR1) in human hepatoma cell lines. Cell Struct Funct 1994, 19:305-313

30. Breitkopf K, Lahme B, Tag CG, Gressner AM: Expression and matrix deposition of latent transforming growth factor beta binding proteins in normal and fibrotic rat liver and transdifferentiating hepatic stellate cells in culture. Hepatology 2001, 33:387-396

31. Flisiak R, Maxwell P, Prokopowicz D, Timms PM, Panasiuk A: Plasma tissue inhibitor of metalloproteinases- 1 and transforming growth factor beta 1: possible non-invasive biomarkers of hepatic fibrosis in patients with chronic B and C hepatitis. Hepatogastroenterology 2002, 49:1369-1372

32. Flisiak R, Prokopowicz D: Transforming growth factor-betal as a surrogate marker of hepatic dysfunction in chronic liver diseases. Clin Chem Lab Med 2000, 38:1129-1131

33. Flisiak R, Pytel-Krolczuk B, Prokopowicz D: Circulating transforming growth factor beta(1) as an indicator of hepatic function impairment in liver cirrhosis. Cytokine 2000, 12:677-681

34. Copin R, Vitry MA, Hanot Mambres D, Machelart A, De Trez C, Vanderwinden JM, Magez S, Akira S, Ryffel B, Carlier Y, Letesson JJ, Muraille E: In situ microscopy analysis reveals local innate immune response developed around Brucella infected cells in resistant and susceptible mice. PLoS Pathog 2012, 8:e1002575

35. Oliveira SC, de Oliveira FS, Macedo GC, de Almeida LA, Carvalho NB: The role of innate immune receptors in the control of Brucella abortus infection: toll-like receptors and beyond. Microbes Infect 2008, 10:1005-1009

36. Oliveira FS, Carvalho NB, Brandao AP, Gomes MT, de Almeida LA, Oliveira SC: Interleukin-1 receptor-associated kinase 4 is essential for initial host control of Brucella abortus infection. Infect Immun 2011, 79:4688-4695

37. Giambartolomei GH, Zwerdling A, Cassataro J, Bruno L, Fossati CA, Philipp MT: Lipoproteins, not lipopolysaccharide, are the key mediators of the proinflammatory response elicited by heat-killed Brucella abortus. J Immunol 2004, 173:4635-4642

38. Ouyang X, Ghani A, Mehal WZ: Inflammasome biology in fibrogenesis. Biochim Biophys Acta 2013, 1832:979-988 